\title{
Characterization of Polyurethane Medical Materials Using Electron Microscopy Technique
}

\author{
J.E. Taylor, ${ }^{(a)}$ P. R. Laity, ${ }^{(a)}$ S.S. Wong, ${ }^{(b)}$ K. Norris, ${ }^{(b)}$ P. Khunkamchoo, ${ }^{(b)}$ A. F. Johnson, ${ }^{(b)}$ \\ M. Cable, ${ }^{(\mathrm{c})}$ V. Chohan, ${ }^{(\mathrm{c})}$ G. Andrews, ${ }^{(\mathrm{c})}$ R. E. Cameron ${ }^{(\mathrm{a})}$ \\ (a) University of Cambridge, Dept. of Materials Science, Cambridge, UK CB2 3QZ \\ (b) University of Leeds, IRC, School of Chemistry, Leeds, UK CB \\ ${ }^{\text {(c) }}$ Ranier Technology, Greenhouse Park Innovation Centre, Cambridge, CB1 5AS
}

The purpose of this research is to determine the relationship among chemistry, microstructure and mechanical performance in thermoplastic polyurethanes (PU) used for medical device applications. Polyurethane materials have been targeted for applications such as coronary assist devices because they are biocompatible with outstanding toughness and flexibility due to random intermixing of the hard (HS) and soft segment (SS) parts of the molecular architecture. A key aspect of the morphology is the interaction between HS and SS parts of the molecular chain since it directly links the polyurethane chemistry to the ultimate properties [1]. Inducing a continuously interconnected HS/SS microstructure can hinder Environmental Stress Cracking (ESC), which is a dominant failure mechanism of implanted PU medical devices.

The chemistry and processing variable were $\mathrm{HS} / \mathrm{SS} \mathrm{wt} \%$ content and post-anneal heat treatments. Structures of the materials were determined at nanometer length scales using a Philips CM300 FEG transmission electron microscopy (TEM). Contrast between HS and SS was induced by heavy element staining of sections cut by cryo-ultramicrotomy. Samples were imaged at Scherzer defocus so comparison among variables could be made. The mesoscale $(>1 \mu \mathrm{m})$ surface structure was examined in-situ as a function of strain using a Philips 2010 Electroscan environmental scanning electron microscopy (ESEM) interfaced to a tensometer. A complimentary study of nanometer structure was performed in-situ by small angle X-ray scattering (SAXS) was performed at the Daresbury synchrotron source.

An interconnected HS microstructure is found in post-annealed samples and samples with greater amounts of $\mathrm{wt} \%$ HS. Figures $1 \mathrm{a}$ and $1 \mathrm{~b}$ show TEM micrographs of $66 \mathrm{wt} \%$ and 71 wt $\%$ HS respectively. Elongated HS domains were found in the sample with a higher HS content compared to more spherical domains observed in sample with a lower HS wt\%. A plot of HS interdomain spacing as a function of $w \mathrm{t} \% \mathrm{HS}$ is shown in figure 2 . These SAXS data suggested that the distance between HS becomes smaller as the HS content is increased. Meso-scale striped features were increasing observed after yield during in-situ ESEM/tensile experiments. These features are shown in figure 3. It is thought that they result from agglomerates of protruding HS, which may be induced by local biaxial stress states [2].

\section{References}

1. Martin et al., J. Appl. Poly. Science 71 (1999) 937.

2. Cerda et al., Nature 419 (2002) 579. 

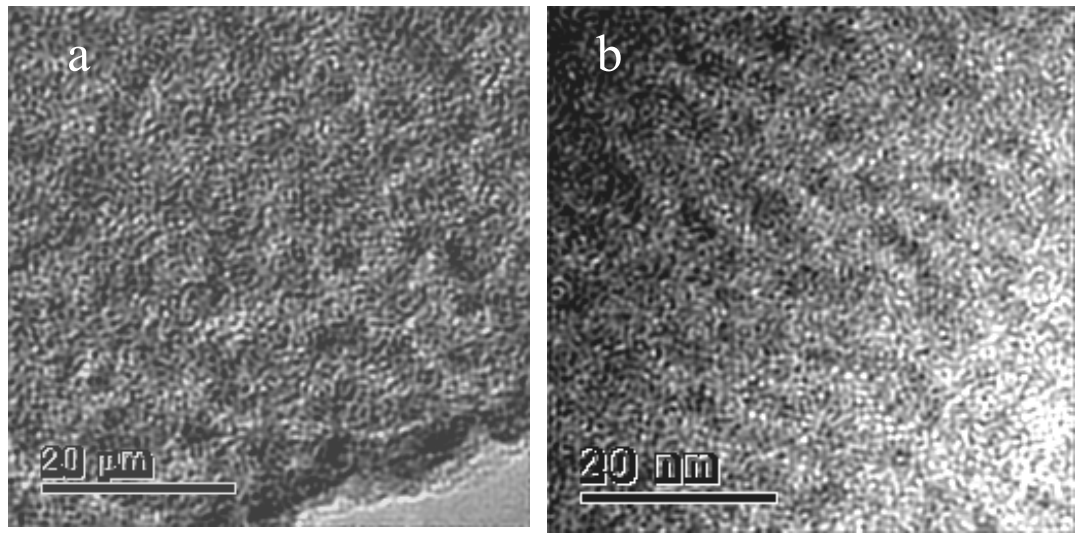

FIG. 1. TEM micrographs of samples containing a. $66 \mathrm{wt} \% \mathrm{HS}$ and b. $71 \mathrm{wt} \% \mathrm{HS}$. HS domains become more elongated as $\mathrm{wt} \% \mathrm{HS}$ is increased.

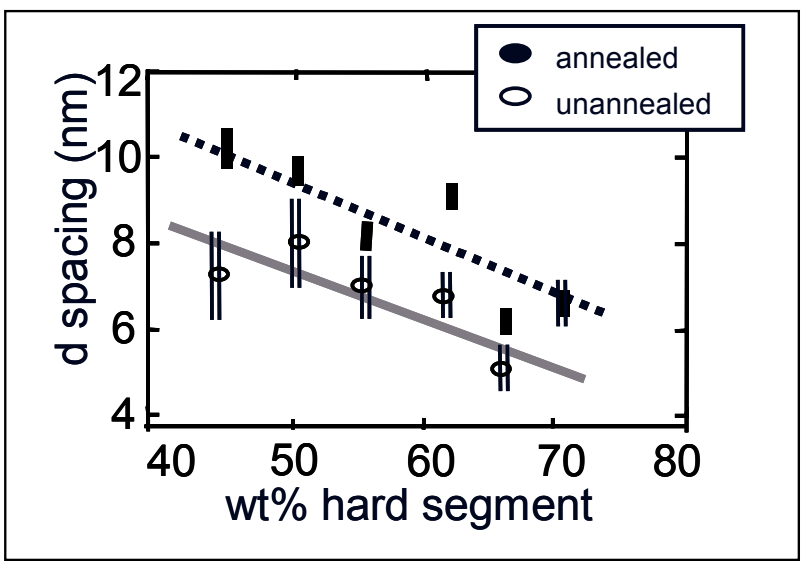

FIG. 2. Plot showing hard segment interdomain spacing vs. wt $\%$ hard segment for annealed and unannealed samples. Data was acquired using SAXS.
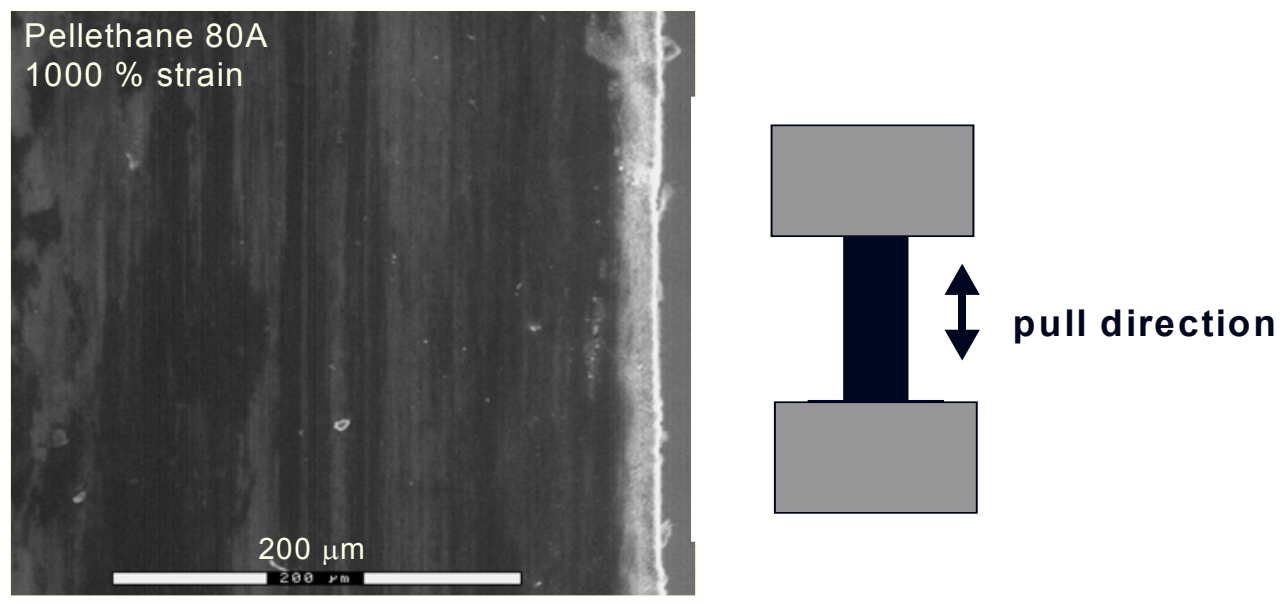

FIG. 3. ESEM micrograph showing strained sample of Pellethane 80A. The striped features may result from local biaxial stress fields. 